\title{
Scaling analysis of galaxy distribution in the Las Campanas Redshift Survey data
}

\author{
T. Kurokawa ${ }^{1,2}$, M. Morikawa ${ }^{1}$, and H. Mouri ${ }^{3}$ \\ 1 Department of Physics, Ochanomizu University, 2-2-1 Otsuka, Bunkyo, Tokyo 112-8610, Japan \\ 2 Institute for Gender Studies, Ochanomizu University, 2-2-1 Otsuka, Bunkyo, Tokyo 112-8610, Japan \\ 3 Meteorological Research Institute, 1-1 Nagamine, Tsukuba 305-0052, Japan
}

Received 14 December 1999 / Accepted 23 November 2000

\begin{abstract}
The Las Campanas Redshift Survey data are used to investigate structures ofthe galaxy number distribution. We construct two volume-limited samples with sizes of $113 \times 113$ and $126 \times 126 h^{-1} \mathrm{Mpc}$, and calculate the second- to ninth-order moments with the count-in-cell method. The galaxy distribution at $\geq 30 h^{-1} \mathrm{Mpc}$ is found to be statistically homogeneous. On the other hand, we find a multifractal scaling at $<30 h^{-1} \mathrm{Mpc}$. From the scaling exponent, we obtain the generalized dimension, which decreases from 2 toward 1 as the order is increased from 2 to 9. Galaxies are known to lie, around voids, in planar structures with filamentary dense regions. The present result indicates that these void-filament structures are predominant up to $30 h^{-1} \mathrm{Mpc}$. Statistically, they appear to be the largest-scale significant structures in the Universe.
\end{abstract}

Key words. cosmology: observations - large-scale structure of Universe - methods: statistical

\section{Introduction}

The galaxy number distribution at large scales $(\gg 10 \mathrm{Mpc})$ is expected to be homogeneous. For example, the X-ray background emission, which originates in distant active galactic nuclei, is isotropic on the sky (e.g. Peebles 1993). Recently, several deep redshift surveys were carried out. The two-point correlation function and power spectrum based on these data indicate that the galaxy distribution actually becomes homogeneous at large scales (e.g. Wu et al. 1999; Martínez 1999). On the other hand, at small scales, the distribution is not homogeneous. Galaxies are distributed roughly on the walls of spherical voids, and show various spatial patterns such as groups, clusters, filaments, and sheets (e.g. Geller \& Huchra 1989). At what scale are these structures predominant? Is there any other structure greater in scale? To answer these questions, we have to study the galaxy distribution in detail.

One promising approach to statistically describe the galaxy distribution is a "multifractal" analysis, which characterizes scaling properties of moments at all the orders (Parisi \& Frisch 1985; Jensen et al. 1985; Halsey et al. 1986; Sect. 3). The distribution is described more precisely than by the standard tools such as the two-point

Send offprint requests to: T. Kurokawa, e-mail: perle@cosmos.phys.ocha.ac.jp correlation function and power spectrum, which are based on the second-order moment alone. Kurokawa et al. (1999) studied galaxy data obtained from the CfA2 survey (Huchra et al. 1999), and found a multifractal scaling at $\leq 20 \mathrm{Mpc}$ (see also, e.g., Domínguez-Tenreiro et al. 1994; Martínez \& Coles 1994). However, because of limitation of the volume size of the CfA2 survey, Kurokawa et al. (1999) were unable to determine the entire scaling range. Of great interest is an analysis of the scaling property at $>20 \mathrm{Mpc}$.

We analyze galaxy data of the Las Campanas Redshift Survey (LCRS). This is the largest complete survey carried out so far and is expected to reach the scale of homogeneity (Shectman et al. 1996, Sect. 2). The volume size of the LCRS data is greater than those in the previous multifractal analyses. Since, however, the LCRS consists of twodimensional slices, we newly define a multifractal analysis on a two-dimensional section of the three-dimensional distribution (Sect. 3). We demonstrate that the galaxy distribution exhibits a multifractal scaling at $<30 \mathrm{Mpc}$ and is homogeneous at $\geq 30 \mathrm{Mpc}$ (Sect. 4). This result is used to discuss the galaxy distribution as a function of scale (Sect. 5). Finally we discuss potential problems of our approach and future prospects (Sect. 6).

Throughout this paper, we use the Hubble constant $H_{0}=100 h \mathrm{~km} \mathrm{~s}^{-1} \mathrm{Mpc}^{-1}$ and the deceleration parameter 
$q_{0}=0.5$. With these parameters, the distance to a galaxy is estimated from its recession velocity in the Local Group frame. We ignore the peculiar velocities of the individual galaxies. They are typically $300 \mathrm{~km} \mathrm{~s}^{-1}$ (Davis \& Peebles 1983), which corresponds to $3 h^{-1} \mathrm{Mpc}$. This scale is less than the scales of our interest $\left(>5 h^{-1} \mathrm{Mpc}\right)$, where we find a multifractal distribution.

\section{Data and sample}

The LCRS provides the largest galaxy sample which has been obtained so far (Shectman et al. 1996). This survey consists of six thin strips on the sky, each of which is $1.5^{\circ}$ in declination, $80^{\circ}$ in right ascension, and made of $1.5^{\circ} \times$ $3^{\circ}$ beams. Thus, the survey volumes are essentially twodimensional slices. There are 26418 galaxies, which were selected from photometry in the $R$-band. Their average redshift is $z \simeq 0.1$. The most distant galaxy lies at $z \simeq 0.4$.

When we analyze the LCRS data, we encounter two problems. First, the LCRS has both upper and lower magnitude limits. They vary from beam to beam. To avoid the effect of this variation, our samples are constructed by applying the narrowest magnitude range to all the beams. Second, in the individual beams, redshifts of some galaxies were not measured. This was due to mechanical constraints of the spectrometer. Since the observed galaxies were selected randomly in the beam, we assigned the inverse of the number fraction of the observed galaxies as a statistical weight to each of the beams.

We extracted volume-limited samples, where the luminosities of the galaxies are above certain thresholds. The $\mathrm{K}$-correction term was set to be $\mathrm{K}(z)=2.5 \log (1+z)$, which provides a good approximation to the $R$-band data of LCRS galaxies (Lin et al. 1996a). We used the LCRS slices $\delta=-3^{\circ}$ and $-12^{\circ}$. The other slices do not generate samples with sufficient numbers of galaxies. Our volumelimited samples contain the largest numbers of galaxies among those with thickness of $4 h^{-1} \mathrm{Mpc}$. The sample from the slice $\delta=-3^{\circ}$ is complete in $16.1 \leq m_{R} \leq 17.1$ and $-21.4 \leq M_{R} \leq-20.3$, contains 173 galaxies, and is $113 \times 113 h^{-1} \mathrm{Mpc}$ in size. Here $m_{R}$ and $M_{R}$ are the apparent and absolute magnitudes in the $R$-band. The sample from the slice $\delta=-12^{\circ}$ is complete in $15.1 \leq m_{R} \leq 17.6$ and $-21.7 \leq M_{R} \leq-20.6$, contains 291 galaxies, and is $126 \times 126 \bar{h}^{-1} \mathrm{Mpc}$ in size. We underline that the volume sizes of these samples are greater than those used in previous multifractal analyses.

In the thickness direction, we applied a Gaussian window function in order to use the samples as twodimensional cuts of the three-dimensional galaxy distribution (e.g. Landy et al. 1996). The full widths at half maximum of the window function are 0.6 and $0.5 h^{-1} \mathrm{Mpc}$, respectively, in the samples for $\delta=-3^{\circ}$ and $-12^{\circ}$. These widths are very small compared to the sample size, $\sim 100 h^{-1} \mathrm{Mpc}$, and have been determined so as to reproduce most clearly the scaling properties of artificial fractal samples with the same number of discrete points as the LCRS data (Sect. 3).
We also studied the three-dimensional CfA2 sample (Huchra et al. 1999). Kurokawa et al. (1999) conducted a multifractal analysis of volume-limited samples with $M_{B(0)} \leq-19.42$ and -20.00 , which contain 358 and 194 galaxies and are $42 \times 42 \times 84$ and $54 \times 54 \times 108 h^{-1} \mathrm{Mpc}$ in size. Their result for the CfA2 galaxies is compared with ours for the LCRS galaxies (Sect. 4). The LCRS and CfA2 observations were made in the $R$ - and $B(0)$-bands. A typical LCRS galaxy is fainter in the $B(0)$-band by $1.8 \mathrm{mag}$ that it is in the $R$-band (Lin et al. 1996a). That is, galaxies in our LCRS samples are less luminous than those in the CfA2 samples. Nevertheless, the luminosity difference does not adversely affect our analysis, because there are no gross differences in the distribution of galaxies at different luminosities (Huchra et al. 1990; Park et al. 1994).

\section{Multifractal analysis on two-dimensional data}

The formal definition of multifractal is not applicable to discrete sets. Hence, for analyses of the galaxy distribution, several different techniques have been proposed. They are reviewed and compared to each other in Ueda (1995). We employ the "count-in-cell" method, which appears to be more straightforward than other methods. Since the LCRS samples are two-dimensional (Sect. 2), the present method is to infer a multifractal distribution in three-dimensional space from the distribution on a twodimensional section.

Suppose that there are $N$ galaxies on a square of size $L$. This square is divided into cells of size $r$. The $q$ th moment $\chi_{q}^{(2)}$ at the scale $r$ is defined as

$\chi_{q}^{(2)}(r)=\sum_{i=1}^{(L / r)^{2}}\left(\frac{n_{i}}{N}\right)^{q} \quad(q \geq 2)$

Here $n_{i}$ is the number of galaxies in the $i$ th cell $(i=$ $\left.1, \ldots,(L / r)^{2}\right)$. The suffix "(2)" indicates that the analysis is conducted on a two-dimensional section. The higherorder moment reflects the structures of the denser regions. We do not study negative-order moments $(q<0)$. If there are vacant cells, they cause divergence of the negative moments.

We plot $\log \left(\chi_{q}^{(2)}\right)$ against $\log (r)$. If the local slope is nearly constant over some range, there is said to be a scaling for that range:

$\chi_{q}^{(2)}(r) \sim r^{(q-1) D_{q}^{(2)}} \quad\left(r_{\min } \leq r \leq r_{\max }\right)$.

Here $D_{q}^{(2)}$ is the generalized dimension. We expect that the generalized dimension in the three-dimensional space is obtained as $D_{q}^{(3)}=D_{q}^{(2)}+1$ (e.g. Meneveau \& Sreenivasan 1991). For example, a sheet-like distribution has $D_{q}^{(2)} \simeq$ 1 and $D_{q}^{(3)} \simeq 2$. Though this method is not effective if $D_{q}^{(3)}<1$, the galaxy distribution always appears to have $D_{q}^{(3)} \geq 1$ (e.g. Domínguez-Tenreiro et al. 1994; Martínez $\&$ Coles 1994; Kurokawa et al. 1999). In a special case of monofractal scaling, all the $D_{q}$ values are equal to the 


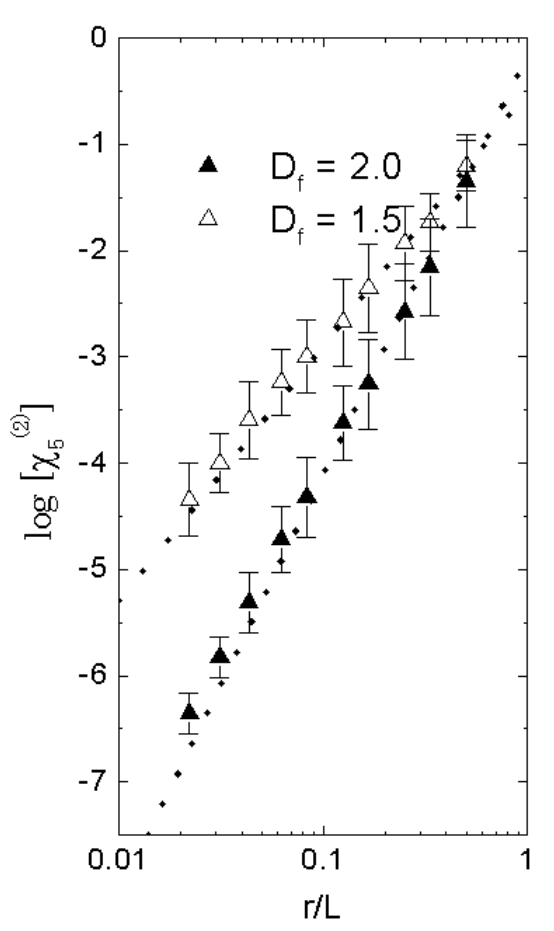

Fig. 1. The fifth-order moments $\chi_{5}^{(2)}$ of Lévy fractal samples with $D_{f}=1.5$ (open triangles) and $D_{f}=2.0$ (filled triangles). The dotted lines represents the relations (2) expected for $D_{q}^{(2)}=D_{f}-1$

fractal dimension $D_{f}$. In the general case of multifractal scaling, $D_{q}^{(3)}$ decreases, i.e., $D_{q}^{(3)} \leq D_{q^{\prime}}^{(3)}$ for $q>q^{\prime}$. The asymptotic value $D_{\infty}^{(3)}$ exists in the limit $q \rightarrow \infty$.

To justify our approach, we analyzed artificial monofractal samples, i.e., "Lévy flight" sets with $D_{f}=1.5$ and 2.0 (e.g. Mandelbrot 1982). The number of the points and the shape of the volume are the same as those of our LCRS sample for $\delta=-3^{\circ}$. We applied the same Gaussian window as the LCRS sample, and calculated the moments $\chi_{5}^{(2)}$. The average and standard deviation over 100 such samples are shown in Fig. 1 . The moments $\chi_{5}^{(2)}$ follow the dotted lines, which represent the relation (2) with $D_{q}^{(2)}=D_{f}-1$. The same result is obtained if the point number, volume shape, and Gaussian window are the same as our LCRS sample for $\delta=-12^{\circ}$. Since the generalized dimensions $D_{q}{ }^{(3)} \simeq 1.5-2.0$ were obtained in previous three-dimensional analyses of the galaxy distribution (e.g. Kurokawa et al. 1999), our method is expected to work well in the following analysis of the LCRS data.

However, in Fig. 1, the moments $\chi_{5}^{(2)}$ at $r / L \ll 0.1$ tend to lie above the dotted lines. They suffer from the finiteness of the point number. When the scale is very small and the number of cells is very large, every cell contains a single point at most. Even if the scale becomes smaller, the number of filled cells remains constant. The moments at these scales are simply proportional to the value $N^{1-q}$. This effect is less significant in the sample with $D_{f}=1.5$. The reason is that a fractal structure has local clusters of points. The effective mean distance among the points is smaller in a fractal set with a smaller $D_{f}$ value (Kurokawa et al. 1999).

\section{Results}

The moments $\chi_{q}^{(2)}$ of the two LCRS samples for $\delta=-3^{\circ}$ and $-12^{\circ}$ are computed with $q=2-9$ and $r / L=1 / 64-$ $1 / 2$. The results for $\delta=-3^{\circ}$ with $q=2,5$, and 9 are shown in Fig. 2 (filled squares). The scale $r$ in units of $h^{-1} \mathrm{Mpc}$ is indicated on the upper axes. The vertical bars on the moments represent the $1 \sigma$ statistical errors evaluated with the "bootstrap resampling" method (Efron 1982; Barrow et al. 1984).

Two scaling ranges are evident in Fig. 2. The transition from the one range to the other occurs within the scale interval of our analysis, which is roughly 0.5 in logarithms. The moments at $\geq 28 h^{-1} \mathrm{Mpc}$ lie on the dotted line, which represents the relation expected for a homogeneous distribution. The moments at $<28 h^{-1} \mathrm{Mpc}$ exhibit another scaling. We apply the least-squares fit to the moments at the scales $7,9,14$, and $19 h^{-1} \mathrm{Mpc}(r / L=1 / 16,1 / 12$, $1 / 8$, and $1 / 6)$. The results are shown by solid lines. Below the scale $5 h^{-1} \mathrm{Mpc}$, the moments do not follow the scaling relation. They suffer from the finiteness of the galaxy number (Sect. 3). The same results have been obtained for the other moments which are not shown in Fig. 2. We have also found a homogeneous distribution at $r \geq 32 h^{-1}$ and another scaling relation at $5<r<32 h^{-1} \mathrm{Mpc}$ in the LCRS sample for $\delta=-12^{\circ}$.

The generalized dimensions $D_{q}^{(2)}$ for the moments at $r<28-32 h^{-1} \mathrm{Mpc}$ are derived from their scaling exponents, i.e., the slopes of the solid lines in Fig. 2. The values of $D_{q}^{(3)}=D_{q}^{(2)}+1$ for $\delta=-3^{\circ}$ (filled squares) and $\delta=-12^{\circ}$ (filled circles) are plotted as a function of $q$ in Fig. 3. We also show the generalized dimensions $D_{q}^{(3)}$ of the CfA2 samples by open circles and squares (Kurokawa et al. 1999). The vertical bars on the data points represent the $1 \sigma$ uncertainties associated with the least-squares fit, which incorporate the statistical errors of the individual moments. Within the $1 \sigma$ uncertainties, the results for the four samples are consistent with each other. The $D_{q}^{(3)}$ value decreases as $q$ increases. Therefore, over the scale and order ranges considered here, the distribution of the LCRS galaxies satisfies the requirement for multifractal scaling.

We have presented only the galaxy moments $\chi_{q}^{(2)}$ with $q \leq 9$. The statistical errors of the higher-order moments are quite large. With an increase of $q$ above 10, the generalized dimension $D_{q}^{(3)}=D_{q}^{(2)}+1$ seems to converge to $D_{\infty}^{(3)} \simeq 1.5$ in both the samples for $\delta=-3^{\circ}$ and $-12^{\circ}$. Their scaling ranges seem to be the same as those for $q \leq 9$. This result is again consistent with that for the CfA2 data, which exhibit $D_{\infty}^{(3)} \simeq 1.4$ (Kurokawa et al. 1999).

In summary, we have found a multifractal distribution at $<30 h^{-1} \mathrm{Mpc}$ and a homogeneous distribution at $\geq 30 h^{-1}$ Mpc. This result has been obtained from both 

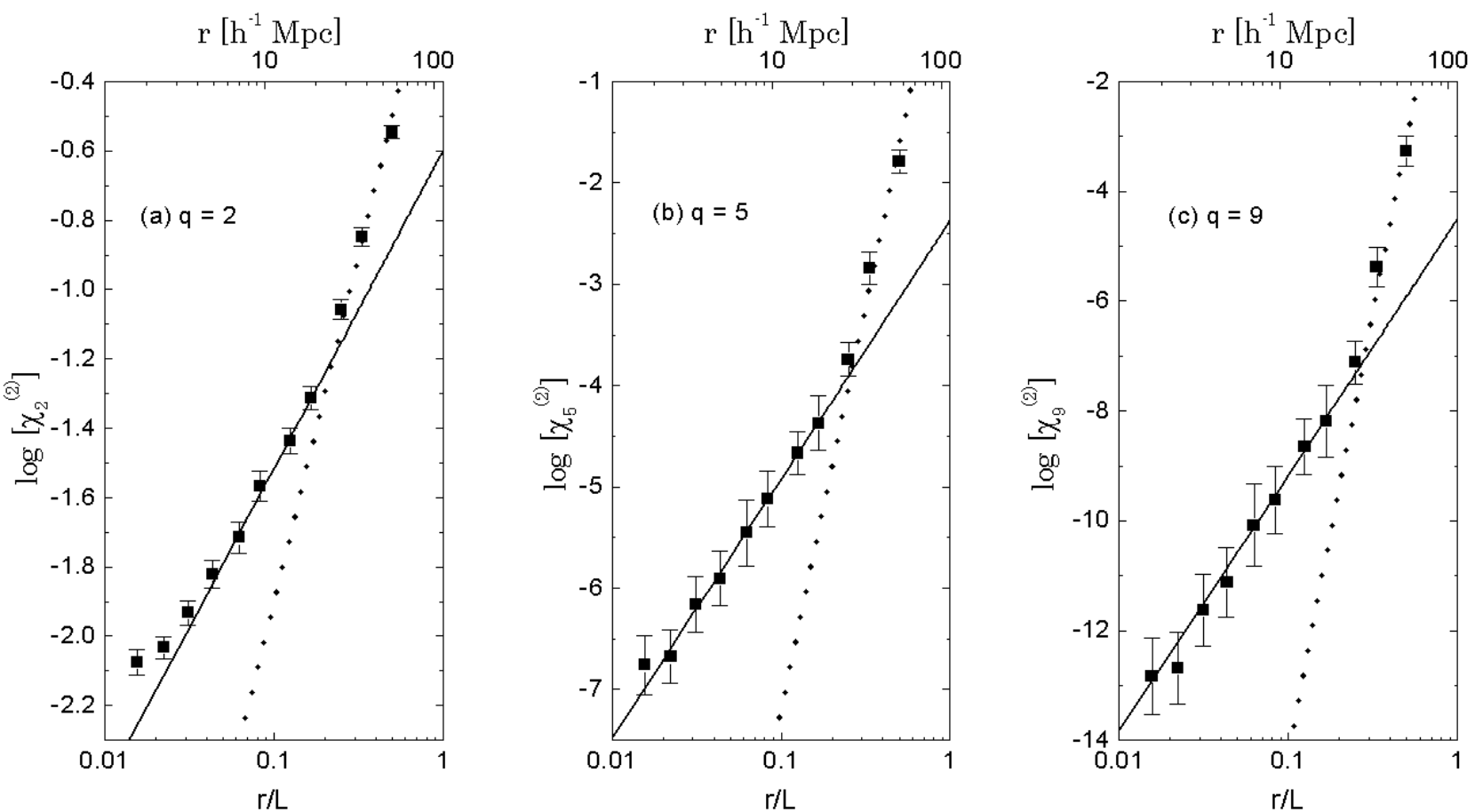

Fig. 2. a) The second-order moments $\chi_{2}^{(2)}$ of the LCRS galaxy sample for $\delta=-3^{\circ}$ (filled squares). The solid line is the result of the least-squares fit at $r=5-20 h^{-1} \mathrm{Mpc}$. The dotted line represents the relation expected for a homogeneous distribution. b) Same as a), but for the fifth-order moments $\chi_{5}^{(2)}$. c) Same as a) but for the ninth-order moments $\chi_{9}^{(2)}$

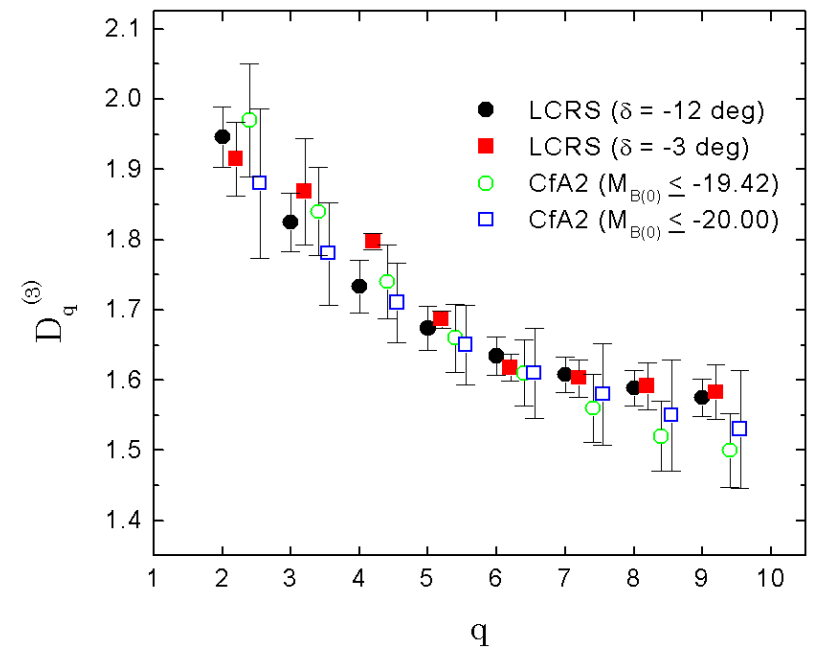

Fig. 3. The generalized dimension $D_{q}^{(3)}=D_{q}^{(2)}+1$ for the LCRS galaxy samples with $\delta=-12^{\circ}$ (filled circles) and with $\delta=-3^{\circ}$ (filled squares). We also show $D_{q}^{(3)}$ for the CfA2 galaxy samples with $M_{B(0)}<-19.42$ (open circles) and with $M_{B(0)}<-20.00$ (open squares)

the LCRS samples for $\delta=-3^{\circ}$ and $-12^{\circ}$, agrees with the result for the CfA2 samples, and hence is expected to be general $^{1}$. Hereafter we separately discuss the multifractal and homogeneous distributions.

\footnotetext{
1 Just before the submission of this paper, we learned that a multifractal analysis of LCRS data was also made by Bharadwaj et al. (1999). They claimed that the galaxy distribution is multifractal at $r=20-80 h^{-1} \mathrm{Mpc}$ and homogeneous at $r \geq 80 h^{-1} \mathrm{Mpc}$. We are unable to compare our result with
}

\section{Discussion}

\subsection{Multifractal distribution at $<30 \mathrm{Mpc}$}

By using the two-point correlation function $\xi(r)$, power spectrum $p(k)$, or other multifractal techniques, it is possible to estimate the $D_{2}^{(3)}$ value (e.g. Guzzo et al. 1991; Martínez \& Coles 1994):

$\xi(r)+1 \sim r^{D_{2}^{(3)}-3} \quad$ and $\quad p(k) \sim k^{-D_{2}^{(3)}}$.

Here $k=2 \pi / r$ is the wavenumber. Table 1 compares the present result with those estimated previously from various galaxy catalogues. They agree roughly in that the $D_{2}^{(3)}$ value is $\simeq 2$ at $r \simeq 5-30 h^{-1} \mathrm{Mpc}$. However, with the results of $D_{2}^{(3)}$ alone, one cannot sufficiently characterize the spatial structures of the galaxy distribution. For example, a Gaussian random field with $p(k) \sim k^{-2}$ yields $D_{2}^{(3)}=2$. To proceed further, higher-order dimensions $D_{q}^{(3)}(q \geq 3)$ such as those obtained in this study are required. They actually show that the galaxy distribution at $<30 h^{-1} \mathrm{Mpc}$ exhibits a multifractal scaling.

With increasing the order $q$, the $D_{q}^{(3)}$ value decreases from 2.0 to 1.5 (Fig. 3). This decrease might be considered small, but it is significant. Since the higherorder moments give more weight to the dense regions,

theirs, because our approach is entirely different. We nevertheless point out that our result is much better consistent with the scaling relation obtained so far from various galaxy catalogues (Sect. 5.1). In addition, Bharadwaj et al. (1999) did not estimate the $D_{q}^{(3)}$ values for the three-dimensional galaxy distribution. 
those $D_{q}^{(3)}$ values are obtained if there are filament-like dense regions within sheet-like structures. The former has $D_{q}^{(3)} \simeq 1$ while the latter has $D_{q}^{(3)} \simeq 2$. Galaxies are known to lie roughly on the walls of spherical voids (e.g. Geller \& Huchra 1989). From the topology of isodensity contours of CfA2 galaxies, Vogeley et al. (1994) found that their distribution forms "walls with holes" or a "filamentary net". Kurokawa et al. (1999) interpreted the multifractal distribution of the CfA2 data as originating in these voidfilament structures. If this interpretation is correct, the present result indicates that the void-filament structures are important up to $30 h^{-1} \mathrm{Mpc}$. The typical size of voids is actually known to be about $30 h^{-1} \mathrm{Mpc}$ (e.g. Peebles 1993).

The above structures can be generated via gravitational instability from primordial random fluctuations (e.g. Jenkins et al. 1998). With $N$-body simulations of cold dark matter models, Doroshkevich et al. (1999) showed richness of sheet-like structures with sizes of 20-30 $h^{-1}$ Mpc. To discriminate between different cosmological models, a comparison of the observed scaling with those of model predictions would be useful.

The observed multifractal scaling is unlikely to extend to the smaller scales $\left(\ll 5 h^{-1} \mathrm{Mpc}\right)$. The value $D_{2}^{(3)} \simeq 2$ estimated for $r \simeq 5-30 h^{-1} \mathrm{Mpc}$ is different from the value $D_{2}^{(3)} \simeq 1.2$ obtained by Guzzo et al. (1991) for $r \leq 3.5 h^{-1}$ Mpc (Table 1 ). These small scales correspond to a typical size of galaxy clusters. They are virialized systems, which originate in processes different to those for the void-filament structures. It is therefore natural to expect different scaling laws between scales above and below $\sim 3.5 h^{-1} \mathrm{Mpc}$.

\subsection{Homogeneous distribution at $\geq 30 \mathrm{Mpc}$}

We have found $D_{q}^{(3)} \simeq 3$ at $\geq 30 h^{-1}$ Mpc. This result is consistent with those in Table 1 . Thus, we conclude that the galaxy distribution at $\geq 30 h^{-1} \mathrm{Mpc}$ is homogeneous (e.g. Wu et al. 1999). The void-filament structures in Sect. 5.1 are the largest-scale spatial structure of the galaxy distribution. Here it should be noted that this conclusion is statistical. The largest void size in the CfA sample is $\simeq 50 h^{-1} \mathrm{Mpc}$. The CfA sample also contains sheet-like structures such as the Great Wall and the Perseus-Pisces Supercluster, which are greater than $100 h^{-1} \mathrm{Mpc}$ in size (e.g. Geller \& Huchra 1989). Such large-scale structures exist in our LCRS samples (Landy et al. 1996). However, the large-scale structures seem to be exceptional or of low amplitude and hence statistically unimportant in the present analyses. The LCRS data yield $\xi(r) \simeq 0$ at $r \simeq 30$ $250 h^{-1}$ Mpc (Tucker et al. 1997; see also Martínez 1999). Kurokawa et al. (1999) studied CfA2 samples containing and not containing the Perseus-Pisces Supercluster, and found no significant diference in the multifractal statistics.
Sylos-Labini et al. (1998) claimed that the galaxy distribution has $D_{2}^{(3)} \simeq 2$ up to $500 h^{-1}$ Mpc. They ignored the K-correction. Scaramella et al. (1998) re-analyzed the same sample with the K-correction, and found a cross-over to homogeneity at several tens of $h^{-1} \mathrm{Mpc}$. When the $\mathrm{K}$-correction is ignored, high-redshift galaxies are estimated to be less luminous. Their number density is underestimated in a volume-limited sample with a certain luminosity threshold. Even if the galaxy distribution is homogeneous, the neglect of the K-correction introduces a spurious structure in the redshift direction. To obtain the $D_{2}^{(3)}$ value, both Sylos-Labini et al. (1998) and Scaramella et al. (1998) used the number of galaxies within the distance $d: N(<d) \sim d^{3-D_{2}^{(3)}}$. This quantity depends directly on the galaxy distribution in the redshift direction, and hence is sensitive to the K-correction (Joyce et al. 1999).

We, on the other hand, have divided a volume-limited sample into square cells, and investigated the number of galaxies in those cells. That is, we have studied the galaxy distribution not only in the redshift direction but also in the perpendicular direction. We expect that our analysis depends only weakly on the K-correction. To demonstrate this, we analyzed the LCRS data for $\delta=-3^{\circ}$ with different K-correction terms. From spectral energy distributions of nearby galaxies, Fukugita et al. (1995) obtained relations between $z$ and the K-correction as a function of morphological type. For spiral to elliptical galaxies, the $\mathrm{K}$-correction term in the $R$-band is $(1.3-5.0) \times \log (1+z)$. We changed the K-correction term in this range, and calculated the moments $\chi_{5}^{(2)}$. The results are shown in Fig. 4 (filled and open diamonds). They are consistent with reference lines, which are the same as in Fig. 2, at least in the multifractal and homogeneous regimes $\left(r>5 h^{-1} \mathrm{Mpc}\right)$.

\section{Concluding remarks}

The scaling property of the galaxy number density has been explored through a multifractal analysis of the LCRS data. Since the LCRS is the largest complete survey carried out so far, our analysis is statistically more significant than the previous multifractal analyses. From the survey slices $\delta=-3^{\circ}$ and $-12^{\circ}$, we have constructed two volumelimited samples. The former is complete in $-21.4 \leq M_{R}$ $\leq-20.3$, contains 173 galaxies, and is $113 \times 113 h^{-1} \mathrm{Mpc}$ in size. The latter is complete in $-21.7 \leq M_{R} \leq-20.6$, contains 291 galaxies, and is $126 \times 126 h^{-1} \mathrm{Mpc}$ in size. The count-in-cell method on a two-dimensional section has been employed. At $\geq 30 h^{-1} \mathrm{Mpc}$, the moments $\chi_{q}^{(2)}$ follow the scaling expected for a homogeneous distribution. On the other hand, from 5 to $30 h^{-1} \mathrm{Mpc}$, we have found a multifractal scaling. The measured generalized dimensions $D_{q}^{(3)}=D_{q}^{(2)}+1$ are consistent with those for the three-dimensional CfA2 galaxy samples. The results for $D_{2}{ }^{(3)}$ are also consistent with those obtained so far from various galaxy catalogues with $\xi(r)$ and $p(k)$.

These results are interpreted as follows. The galaxy distribution at $\geq 30 h^{-1} \mathrm{Mpc}$ is homogeneous. From 5 
Table 1. Estimates of the second-order generalized dimension $D_{2}^{(3)}$

\begin{tabular}{lcccc}
\hline \hline sample & $D_{2}^{(3)}$ & scale range $^{a}$ & method $^{b}$ & reference $^{-1} \mathrm{Mpc}$ \\
\hline Perseus-Pisces & $1.25 \pm 0.10$ & $(1)<r \leq 3.5$ & $\xi(r)+1$ & 1 \\
CfA1 & $1.23 \pm 0.04$ & $0.1<r \leq 5$ & $\xi(r)$ & 2 \\
\hline Perseus-Pisces & $2.21 \pm 0.06$ & $3.5 \leq r \leq 25-30$ & $\xi(r)+1$ & 1 \\
CfA1 & $\simeq 2$ & $1<r \leq 30$ & $(\xi(r)+1) n$ & 3 \\
CfA1 & $\simeq 2$ & $(1.5) \leq r \leq 20-30$ & multifractal $^{c}$ & 4 \\
CfA2 $\left(M_{B(0)} \leq-19.42\right)$ & $1.95 \pm 0.08$ & $(5) \leq r \leq(21)$ & multifractal $^{d}$ & 5 \\
CfA2 $\left(M_{B(0)} \leq-20.00\right)$ & $1.89 \pm 0.06$ & $(7) \leq r \leq(27)$ & multifractal $^{d}$ & 5 \\
IRAS QDOT & 2.25 & $(1) \leq r<10$ & multifractal $^{c}$ & 6 \\
LCRS & $1.8 \pm 0.1$ & $5<r<30$ & $p(k)$ & 7 \\
LCRS & $\simeq 2$ & $(4)<r \leq 20-30$ & $\xi(r)+1$ & 8 \\
LCRS $\left(\delta=-3^{\circ}\right)$ & $1.92 \pm 0.06$ & $(5)<r<28$ & multifractal $^{d}$ & 9 \\
LCRS $\left(\delta=-12^{\circ}\right)$ & $1.96 \pm 0.05$ & $(5)<r<32$ & multifractal $^{d}$ & 9 \\
\hline Perseus-Pisces & $\simeq 3$ & $25-30 \leq r<(70)$ & $\xi(r)+1$ & 1 \\
CfA1 & $\simeq 3$ & $20-30 \leq r \leq(40)$ & multifractal $^{c}$ & 4 \\
IRAS QDOT & 2.77 & $10<r \leq(50)$ & multifractal $^{c}$ & 6 \\
LCRS $\left(\delta=-3^{\circ}\right)$ & $\simeq 3$ & $28 \leq r \leq(57)$ & multifractal $^{d}$ & 9 \\
LCRS $\left(\delta=-12^{\circ}\right)$ & $\simeq 3$ & $32 \leq r \leq(63)$ & multifractal $^{d}$ & 9 \\
Abell Clusters & $2.93 \pm 0.15$ & $(70) \leq r \leq(300)$ & $N(<d)$ & 10 \\
ESO Slice Project & $3.08 \pm 0.18$ & $(300) \leq r<(500)$ & $N(<d)$ & 10 \\
\hline
\end{tabular}

Notes. $(a)$ the value in parentheses is the analysis limit, which is determined by the volume or galaxy number of the sample; $(b)$ $\xi(r)$ is the two-point correlation function, $n$ is the mean number density, $p(k)$ is the power spectrum, and $N(<d)$ is the number of galaxies within the distance $d ;(c)$ the density reconstruction method; $(d)$ the count-in-cell method.

References. (1) Guzzo et al. 1991; (2) Davis \& Peebles 1983; (3) Lemson \& Sanders 1991; (4) Domínguez-Tenreiro et al. 1994; (5) Kurokawa et al. 1999; (6) Martínez \& Coles 1994; (7) Lin et al. 1996b; (8) Amendola \& Palladino 1999; (9) this work; (10) Scaramella et al. 1998.

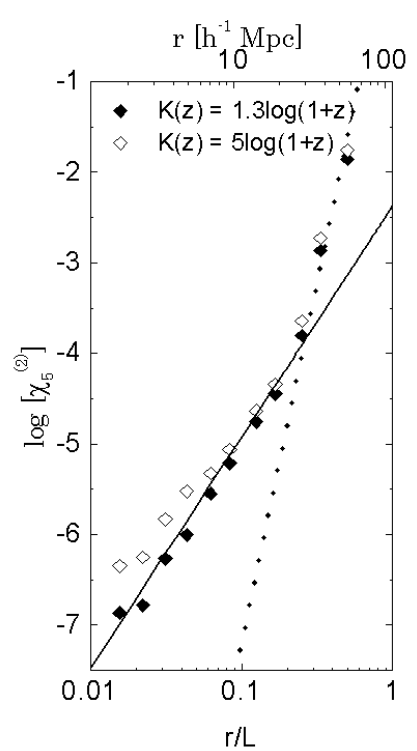

Fig. 4. The fifth-order moments $\chi_{5}^{(2)}$ of the LCRS galaxy samples for $\delta=-3^{\circ}$ with $K(z)=1.3 \log (1+z)$ (filled diamonds) and with $K(z)=5.0 \log (1+z)$ (open diamonds). The reference lines are the same as in Fig. 4

to $30 h^{-1} \mathrm{Mpc}$, void-filament structures are predominant. Statistically, they are the largest-scale spatial structures of galaxy distribution. The multifractal scaling is unlikely to extend to small scales ( $\ll 5 h^{-1} \mathrm{Mpc}$ ), where the structures are expected to be virialized.

Since the LCRS samples are two-dimensional, we have been unable to apply a three-dimensional analysis. The galaxy distribution in three-dimensional space has been inferred from the distribution in two-dimensional sections. However, the relation $D_{q}^{(3)}=D_{q}^{(2)}+1$ is not applicable to all cases, although this relation is valid for Lévy flight sets that have nearly the same distribution as the galaxies. Moreover, there are structures much larger than $30 h^{-1} \mathrm{Mpc}$ in size. Our two-dimensional LCRS samples might have missed such structures by chance, or our multifractal method might be unable to detect them. For example, the two-dimensional power spectrum of the LCRS data exhibits a strong peak at $\simeq 100 h^{-1} \mathrm{Mpc}$ (Landy et al. 1996). We have ascertained that the present results for the two-dimensional LCRS data are consistent with previous analyses for the three-dimensional data (e.g. the CfA2 sample; Kurokawa et al. 1999), but it is important to confirm our results by analyzing a three-dimensional galaxy sample that is as deep as the present LCRS samples with various statistical methods. We hope that on-going redshift surveys such as the Sloan Digital Sky Survey will soon provide such galaxy data. 
Acknowledgements. This paper is in part the result of research of T. Kurokawa toward fulfillment of the requirements of the $\mathrm{Ph}$. D. degree at the Ochanomizu University. T. Kurokawa thanks O. Iguchi, I. Joichi, M. Obara, T. Okamura, Y. Sota, E. Takayama, and A. Yoshisato for interesting discussion. We also thank M. Lachièze-Ray for useful comments.

\section{References}

Amendola, L., \& Palladino, E. 1999, ApJ, 514, L1

Barrow, J. D., Bhavsar, S. P., \& Sonoda, D. H. 1984, MNRAS, $210,19 \mathrm{P}$

Bharadwaj, S., Gupta, A. K., \& Seshadri, T. R. 1999, A\&A, 351,405

Davis, M., \& Peebles, P. J. E. 1983, ApJ, 267, 465

Domínguez-Tenreiro, R., Gómez-Flechoso, M. A., \& Martínez, V. J. 1994, ApJ, 424, 42

Doroshkebich, A. G., Múller, V., Retzlaff, J., \& Turchaninov, V. 1999, MNRAS, 306, 575

Efron, B. 1982, The Jackknife, the Bootstrap, and Other Resampling Plans (S.I.A.M, Philadelphia)

Fukugita, M., Shimasaku, K., \& Ichikawa, T. 1995, PASP, 107, 945

Geller, M. J., \& Huchra, J. P. 1989, Science, 246, 897

Guzzo, L., Iovino, A., Chincarini, G., Giovanelli, R., \& Haynes, M. P. 1991, ApJ, 382, L5

Halsey, T. C., Jensen, M. H., Kadanoff, L. P., Procaccia, I., \& Shraiman, B. I. 1986, Phys. Rev. A, 33, 1141

Huchra, J. P., Geller, M. J., de Lapparent, V., \& Corwin, H. G. 1990, ApJS, 72, 433

Huchra, J. P., Vogeley, M. S., \& Geller, M. J. 1999, ApJS, 121, 287

Jenkins, A., Frenk, C. S., Pearce, F. R., et al. 1998, ApJ, 499, 20

Jensen, M. H., Kadanoff, L. P., Libchaber, A., Procaccia, I., \& Stavans, J. 1985, Phys. Rev. Lett., 55, 2798
Joyce, M., Montuori, M., Sylos Labini, F., \& Pietronero, L. 1999, A\&A, 344, 387

Kurokawa, T., Morikawa, M., \& Mouri, H. 1999, A\&A, 344, 1

Landy, S. D., Shectman, S. A., Lin, H. L., et al. 1996, ApJ, 456, L1

Lemson, G., \& Sanders, R. H. 1991, MNRAS, 252, 319

Lin, H., Kirshner, R. P., Shectman, S. A., et al. 1996a, ApJ, 464, 60

Lin, H., Kirshner, R. P., Shectman, S. A., et al. 1996b, ApJ, 471,617

Mandelbrot, B. B. 1982, The Fractal Geometry of Nature (Freeman, San Francisco)

Martínez, V. J. 1999, Science, 284, 445

Martínez, V. J., \& Coles, P. 1994, ApJ, 437, 550

Meneveau, C., \& Sreenivasan, K. R. 1991, J. Fluid Mech., 224, 429

Parisi, G., \& Frisch, U. 1985, On the singularity structure of fully developed turbulence, in ed. M. Ghil, R. Benzi, \& G. Parisi, Turbulence and Predictability in Geophysical Fluid Dynamics (North-Holland, Amsterdam), 84

Park, C., Vogeley, M. S., Geller, M. J., \& Huchra, J. P. 1994, ApJ, 431, 569

Peebles, P. J. E. 1993, Principles of Physical Cosmology (Princeton Univ. Press)

Scaramella, R., Guzzo, L., Zamorani, G., et al. 1998, A\&A, 334,404

Shectman, S. A., Landy, S. D., Oemler, A., et al. 1996, ApJ, 470, 172

Sylos-Labini, F., Montuori, M., \& Pietronero, L. 1998, Phys. Rep., 293, 61

Tucker, D. L., Oemler, A., Kirshner, R. P., et al. 1997, MNRAS, 285, L5

Ueda, H. 1995, PASJ, 47, 389

Vogeley, M. S., Park, C., Geller, M. J., Huchra, J. P., \& Gott III, J. R. 1994, ApJ, 420, 525

Wu, K. K. S., Lahav, O., \& Rees, M. J. 1999, Nature, 397, 225 